\title{
In vitro characterization of mucoadhesive polysaccharide polymers tablets fabricated using FTIR press
}

\author{
Ashwini Kumar ${ }^{1}$ (D), Sudhanshu Kumar Bharti² (D), Awanish Kumar ${ }^{1}$ (D) \\ 'National Institute of Technology, Department of Biotechnology, Raipur (Chhattisgarh), India \\ ${ }^{2}$ Patna University, Department of Biochemistry, Patna (Bihar), India
}

ORCID IDs of the authors: A.K. 0000-0002-4696-4652; S.K.B. 0000-0001-9925-5553; A.K. 0000-0001-8735-479X

Cite this article as: Kumar, A., Kumar Bharti, S., \& Kumar, A. (2020). In vitro characterization of mucoadhesive polysaccharide polymers tablets fabricated using FTIR press. İstanbul Journal of Pharmacy, 50 (2), 79-82.

\begin{abstract}
Background and Aim: Buccal/sublingual drug delivery is gradually becoming one of the most experimented routes for alternate drug delivery. The major advantage of both routes is their high vascularity that allows a substantial permeation of drugs into systemic circulation. Mucoadhesive biopolymers are the mainstay of a transmucosal drug delivery system.

Methods: We formulated blank tablets of explored mucoadhesive biopolymers (sodium alginate, carboxymethyl cellulose, hydroxyl propyl methyl) cellulose using a combination of two at a time. The novelty of this script lies in the formation of tablets using the FTIR hydraulic press, as opposed toa conventional tablet punching machine, at two different pressures. The tablets were subjected to basic characterizations of polymeric interaction, hardness, and swelling behaviour.

Results: An interaction analysis using XRD revealed a good interaction between the polymers. HC-300 and AC-300 were found to be the hardest among the tablets formulated. In terms of swelling behaviour, AC-200 and HA-300 displayed the best swelling as compared to other combinations.

Conclusion: In the absence of a conventional tablet punching machine, we fabricated swellable biopolymeric tablets using the regular $\mathrm{KBr}$ hydraulic press that comes as an accessory with the FTIR instrument. These tablets can possible be used for delivering drugs through buccal mucosa.
\end{abstract}

Keywords: Biopolymer, FTIR press, tablets, drug delivery, transmucosal

\section{INTRODUCTION}

Buccal and sublingual mucosae have become sites of great interest for local and systemic drug delivery. They are gradually becoming the preferred routes since they can bypass the first-pass metabolism usually encountered through the oral route. Secondly, drugs that are administered parenterally encounter poor patient compliance (Vila, Tardelli, Chaud, Tubino, \& Balcão, 2014). For those drugs, this route can completely bypass the pain and local site morbidity. The basic anatomy of buccal mucosa consists of an epithelial layer (stratified squamous epithelia) which overlays a layer of connective tissue known as lamina propria. The buccal epithelium is approximately 50 cell layers thick and non-keratinized. The buccal mucosa is approximately $500-800 \mu m$ thick with a surface area of around $50 \mathrm{~cm}^{2}$. Numerous tiny blood vessels are present in lamina propria that drain into the jugular vein providing a direct entry point for drugs delivered through the buccal mucosal route. The presence of saliva is key to mucoadhesion and degradation of the formulation. The viscosity and mucoadhesion support rendered by saliva is attributed to the presence of glycoprotein mucin. The drug permeation via the buccal route takes place mainly by the paracellular and transcellular route (Barua et al., 2016; Boddupalli, Mohammad, Nath, \& Banji, 2010). The sublingual area, currently the most widely utilized oral transmucosal 
site, is more vascular as compared to the buccal mucosa. Being thinner and non-keratinized, it provides better permeability to drugs (Sudhakar, Kuotsu, \& Bandyopadhyay, 2006).

Patient acceptability has been one of the major advantages of buccal/sublingual mucosae apart from the technical advantage of bypassing the first-pass metabolism. Various formulations such as mucoadhesive tablets, sprays, gels, and patches have been formulated to date (Barua et al., 2016; Mura, Cirri, Mennini, Casella, \& Maestrelli, 2016). The most recent work on mucoadhesive buccal tablets reports freeze-dried formulations for prilocaine and lidocaine as local anesthetics for dental procedures (Favacho et al., 2020). Mucoadhesive polymers have been the mainstay of drug delivery formulation through mucosal routes. Some widely explored polymers include hydroxypropyl methylcellulose (HPMC), sodium carboxymethyl cellulose (NaCMC), alginate (ALG), chitosan $(\mathrm{CH})$, and xanthan gum (XG) (Shridhar, Manohar, \& Bhanudas, 2013). Favacho et al. (2020) have used Pullulan as the mucoadhesive polymer to formulate the mucoadhesive tablet.

The novelty of the work presented in this script lies in the fabrication of biopolymeric tablets from ALG, NaCMC, and HPMC mucoadhesive biopolymers using the non-conventional simple in-house available FTIR KBr hydraulic press at two different pressures. Utilization of an FTIR press to the polysaccharide tableting was an interesting and ingenious idea that was further subjected to hardness testing, polymeric interactions, and swelling behaviour in simulated salivary fluid-based solid agar base. Our tablets displayed mutual interaction among polymers and significant swelling behaviour while significantly low hardness as compared to the regularly reported values.

\section{MATERIALS AND METHODS}

\section{Materials}

Sodium alginate (ALG; CAS No. 9005383) was procured from SRL Chemicals, India. Hydroxypropyl methyl cellulose (HPMC; CAS No. 9004653) was procured from Molychem, Mumbai, India while sodium carboxy methyl cellulose (NaCMC; CAS No. 9004324) was procured from Fisher Scientific, Mumbai, India. All other chemicals mentioned were procured from HiMedia, India.

\section{Tablet formation}

Since we did not have a regular tablet punching machine, for this study we utilized the $\mathrm{KBr}$ press machine that is an important associated part of the FTIR spectrophotometer. The $\mathrm{KBr}$ press was Metrex made (Figure 1). The blank or unloaded tablets were formulated in the combination mixture of (ALG + $\mathrm{NaCMC}),(\mathrm{ALG}+\mathrm{HPMC})$ and (NaCMC + HPMC).

\section{Hardness test}

The tablets were subjected to hardness using the Pfizer hardness tester. Three tablets of each combination were tested, and an average was taken.

\section{Ingredient interaction study}

The interaction between ingredients was visualized by X-ray diffraction (XRD) using a PANalyticalX'Pert instrument (Malvern

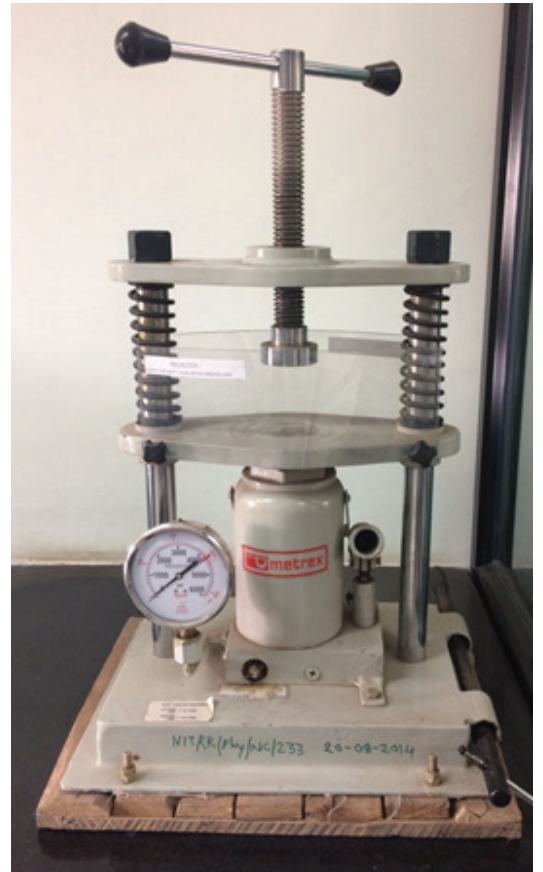

Figure 1. Camera image of the KBr press used to form tablets.

Panalytical, UK). The XRD graphs were plotted for each individual polymer and each combination formulation.

\section{In vitro swelling study}

Formulations targeted for specific routes need to be tested in simulated conditions. Therefore, the swelling behaviour of formulated tablets was analysed using a simulated salivary fluid. The simulated salivary fluid was formulated in accordance with the formula suggested by Koland et al. (Koland, Vijayanarayana, Charyulu, \& Prabhu, 2011). Briefly, it consisted of disodium hydrogen phosphate $(2.38 \mathrm{~g} / \mathrm{L})$, potassium dihydrogen phosphate $(0.19 \mathrm{~g} / \mathrm{L})$, and sodium chloride $(8 \mathrm{~g} / \mathrm{L})$. The $\mathrm{pH}$ was maintained at 6.75. Agar plates were formed by dissolving $2 \%$ agar (w/v) in the simulated salivary fluid. The tablets, after being weighed for the initial weight ( 0 hour), were kept in agar plates at $37^{\circ} \mathrm{C}$ in the incubator. The weight of the tablets was measured at an interval of one hour for five consecutive hours.

\section{RESULTS}

\section{Tablet formulation}

Tablets were formed at two different pressures of 200 psi and 300 psi. The tablets were eight $\mathrm{mm}$ in diameter with a thickness of $2 \pm 0.4 \mathrm{~mm}$. The tablets were uniform in shape and size (Figure 2a).

\section{Hardness test}

As stated in the previous section, since we did not have a regular tablet punching machine, the tablets formed from the hydraulic press of the FTIR spectrophotometer lacked the hardness reported usually. The mean hardness of each formulation is shown in Figure 2b. AC 300 and HC 300 displayed the best hardness among the fabricated tablets. 


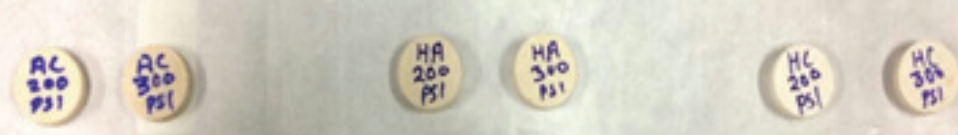

(2a)

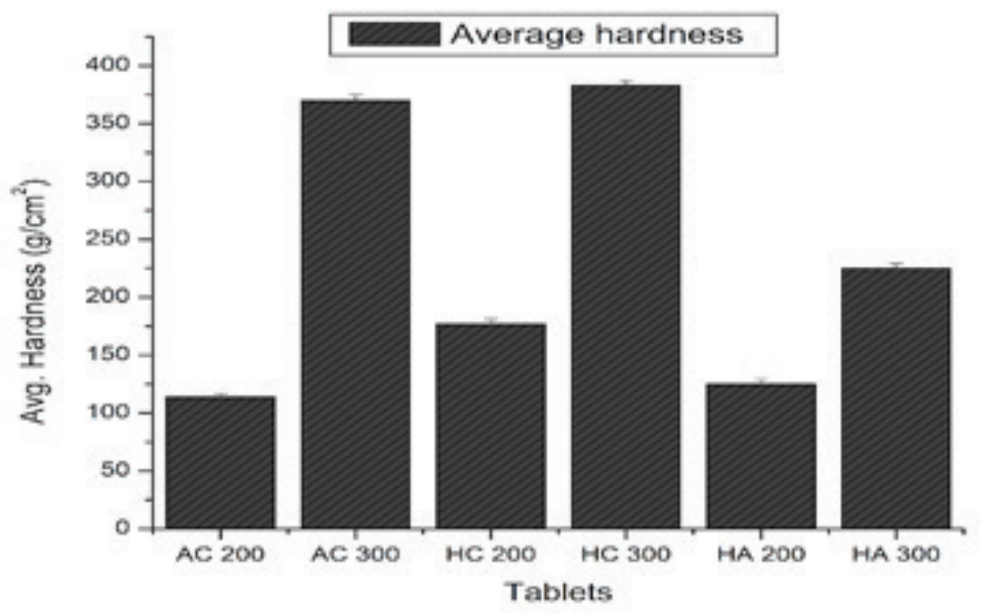

(2b)

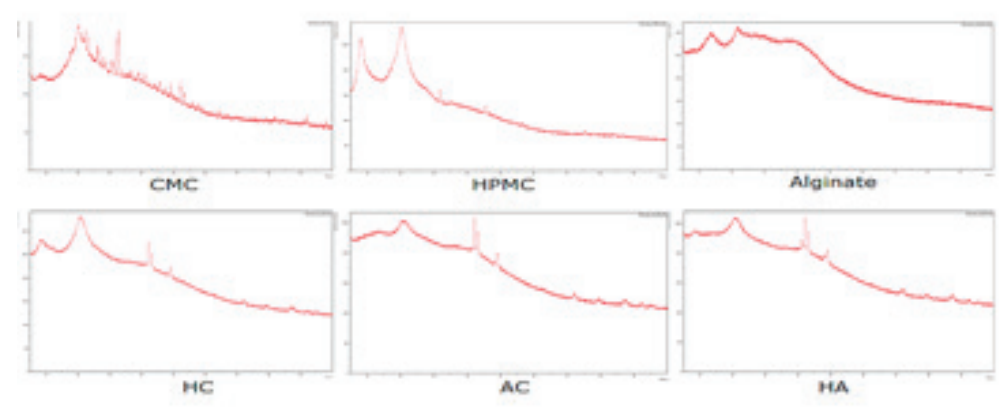

Interaction between polymers as detected by XRO

Figure 2. Camera image of tablets (2a); Hardness of tablets (2b); Interaction between polymers (2c).

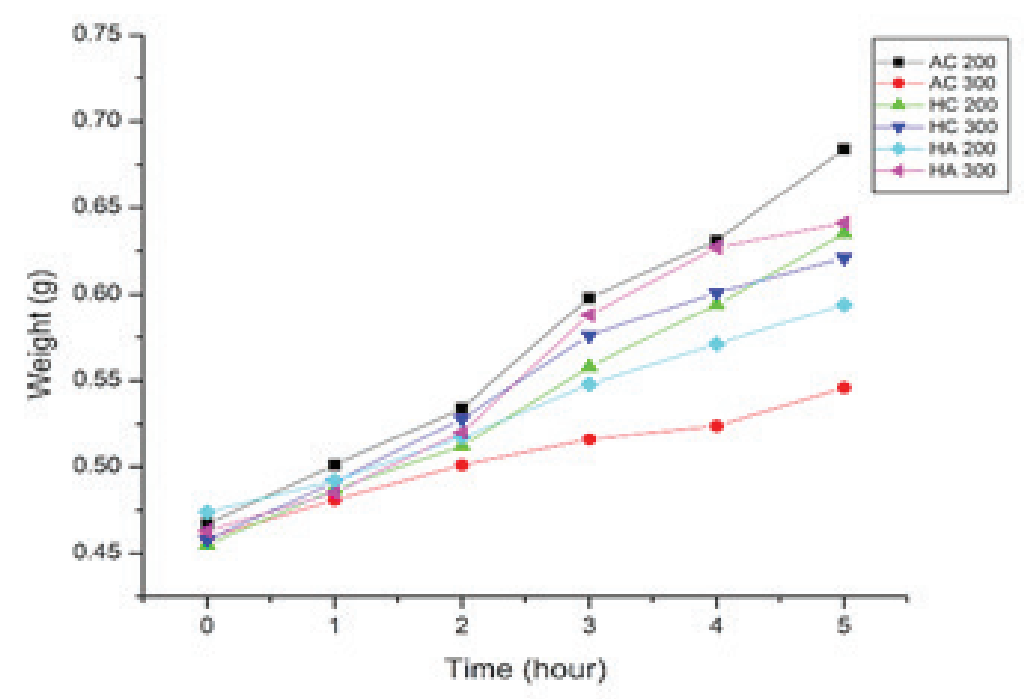

(3a)

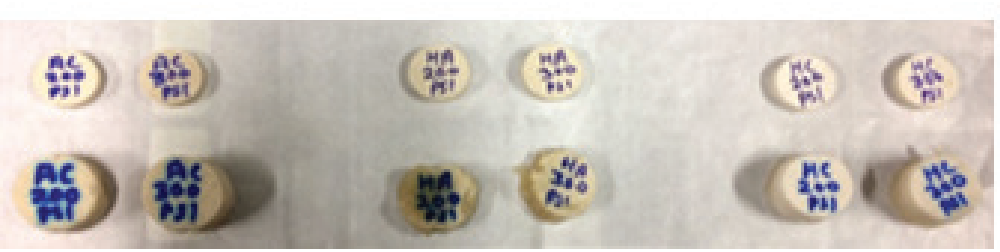

Figure 3. Swelling behaviour of tablets (3a); Camera image before and after swelling (3b). 


\section{Interaction study}

The ingredient interaction ensures bonding and stability of the formulation. Our tablets, on being subjected to XRD analysis, revealed a significant change in the peaks as compared to the peaks of individual polymers that divulge the presence of molecular interactions among polymers. The interaction results are shown in Figure 2c.

\section{In-vitro swelling study}

The swelling of buccal tablets is an important parameter since a drug will be released upon swelling of the formulation. Swelling results in weakening of interactions among polymers would lead to sustained release of the drug. This study revealed that the best swelling is shown by tablets namely AC 200 and HA 300. The graph representing the swelling behaviour of tablets displayed in Figure 3a. Figure 3b shows a comparative image of tablets before and after the swelling study.

\section{DISCUSSION}

Buccal/sublingual mucosal routes are gradually becoming one of the most explored alternative routes for drug delivery. These sites are easily accessible and highly vascular. Drugs that display extensive first-pass metabolism orally or that are administered at a very high dose are usually considered for this route. The delivery systems for these routes consist of mucoadhesive polymers as the primary carriers. The first and foremost requisition for polymers to be considered for being a carrier in such a delivery system is being mucoadhesive. Secondly, the biopolymers should be biocompatible or non-immunogenic to the specific site in particular and the human system in general. Biopolymeric tablets provide an easy and compatible way to deliver drugs through the buccal/sublingual route since they stay for long and are considered good for a sustained drug release (Sudhakar et al., 2006).

This study was aimed at proving that the formation of tablets using three established mucoadhesive biopolymers and a FTIR hydraulic press could be a feasible alternative to a tablet punching machine. The tablets formed underwent basic essential characterizations such as hardness, interaction, and swelling behaviour. The study revealed that AC 200 and HA 300 tablets displayed the best swelling while HC 300 and AC 300 displayed the best hardness. We agree that we have not used any tablet binder that is commonly applied, but the use of FTIR press can result in tablet formation using pressure and these tablets displayed swelling without significant fragility in a simulated salivary fluid. It must be understood that for transbuc$\mathrm{cal} /$ sublingual drug delivery, the formulation cannot be kept for more than 2 hours (for patient compliance related to eating and drinking) if the formulation is not fast disintegrating and degradable. Since this is a preliminary study, better optimization is definitely required to formulate tablets for transbuccal/ sublingual drug delivery so that the maximum amount of drug can be delivered within 2 hours through the buccal mucosa. The usefulness of the FTIR hydraulic press should be consid- ered as a feasible alternative for tablet formation. Further modifications and characterizations of these biopolymeric tablets can be extended to formulate a better oral transmucosal drug delivery system.

\section{Peer-review: Externally peer-reviewed.}

Author Contributions: Conception/Design of Study- A.K., A.K.; Data Acquisition- A.K., A.K.; Data Analysis/Interpretation- A.K., S.K.B., A.K.; Drafting Manuscript- A.K., S.K.B.; Critical Revision of Manuscript- A.K.; Final Approval and Accountability- A.K., S.K.B., A.K.; Technical or Material Support- A.K., S.K.B., A.K.; Supervision- A.K.

Conflict of Interest: The authors have no conflict of interest to declare.

Financial Disclosure: Authors declared no financial support.

Acknowledgements: The authors acknowledge the contribution of NIT Raipur and Patna University for providing space and resources to complete this short research work.

\section{REFERENCES}

- Barua, S., Kim, H., Jo, K., Seo, C. W., Park, T. J., Lee, K. B. ... Lee, J. (2016). Drug delivery techniques for buccal route: Formulation strategies and recent advances in dosage form design. Journal of Pharmaceutical Investigation, 46, 593-613. https://doi. org/10.1007/s40005-016-0281-9.

- Boddupalli, B. M., Mohammad, Z. N. K., Nath, R. A., \& Banji, D. (2010). Mucoadhesive drug delivery system - an overview. Journal of Advanced Pharmaceutical Technology and Research, 1(4), 381-387. https://doi.org/10.4103/0110-5558.76436

- $\quad$ Favacho, H. A. S., do Couto, R. O., Duarto, M. P. F., Peixoto, M. P. G., Lopez, R. F. V., Pedrazzi, V, de Gaitani, C. M., \& de Freitas, O. (2020). Synergy between surfactants and mucoadhesive polymers enhances the transbuccal permeation of local anesthetics from freeze-dried tablets. Material Science and Engineering: C, 108, 110373. https://doi.org/10.1016/j.msec.2019.110373.

- Mura, P., Cirri, M., Mennini, N., Casella, G., \& Maestrelli, F. (2016). Polymeric mucoadhesive tablets for topical or systemic buccal delivery of clonazepam: Effect of cyclodextrin complexation. Carbohydrate Polymers, 152, 755-763. https://doi.org/10.1016/j. carbpol.2016.07.075

- $\quad$ Koland, M., Vijayanarayana, K., Charyulu, R. N., \& Prabhu, N. (2011). In vitro and in vivo evaluation of chitosan buccal films of ondansetron hydrochloride. International Journal of Pharmaceutical Investigation, 1(3), 164-171. https://doi.org/10.4103/2230-973X.85967 Shridhar, G. S., Manohar, S. D., \& Bhanudas, S. R. (2013). Mucoadhesive buccal drug delivery: An overview. Journal of Advanced Pharmacy Education and Research,3(4), 319-332.

- Sudhakar, Y., Kuotsu, K., \& Bandyopadhyay, A. K. (2006). Buccal bioadhesive drug delivery - A promising option for orally less efficient drugs. Journal of Controlled Release, 114, 15-40. https:// doi.org/10.1016/j.jconrel.2006.04.012

- Vila, M. M., Tardelli, E. R., Chaud, M. V., Tubino, M., \& Balcão, V. M. (2014). Development of a buccal mucoadhesive film for fast dissolution: Mathematical rationale, production and physicochemical characterization, Drug Delivery, 21(7), 530-539. https://doi.org /10.3109/10717544.2013.851301. 